\title{
Intrahepatic Infusion Procedure
}

National Cancer Institute

\section{Source}

National Cancer Institute. Intrahepatic Infusion Procedure. NCI Thesaurus. Code C15731.

Administration of a fluid form of the drug via a blood vessel into the liver over the sustained period of time. 